\title{
British Thoracic Society adult community acquired pneumonia audit 2009/10
}

\author{
Wei Shen Lim, ${ }^{1}$ Mark Woodhead, ${ }^{2}$ On behalf of the British Thoracic Society
}

\begin{abstract}
See Editorial, p 460
- Additional tables are published online only. To view these files please visit the journal online (http://thorax.bmj. com).

${ }^{1}$ Department of Respiratory Medicine, Nottingham City Hospital, Nottingham, UK ${ }^{2}$ Department of Respiratory Medicine, Manchester Royal Infirmary, Manchester, UK

Correspondence to Wei Shen Lim, Respiratory Medicine, Nottingham University Hospitals NHS Trust, City Campus, Nottingham NG5 1PB, UK;

weishen.lim@nuh.nhs.uk
\end{abstract}

Accepted 23 February 2011 Published Online First 17 April 2011

\begin{abstract}
Background The updated British Thoracic Society (BTS) Guidelines for the management of Community Acquired Pneumonia (CAP) in adults was published in October 2009. In conjunction with the Guidelines, the first national BTS audit of adult CAP was conducted.

Methods An audit tool was developed as part of the Guidelines. Members of the BTS were invited to participate in the audit capturing data relating to acutely ill adults admitted to hospitals in the UK and treated for CAP within the period 1 December 2009 and 31 January 2010. Data entry using the web-based audit tool closed in May 2010.
\end{abstract}

Results Of 2749 submissions from 64 institutions; 8 were excluded due to inconsistent data. The mean age of patients was 71 years (range 16-105 years). The CURB65 score was 0 to 1 in 40\% of patients, 2 in 30\% and 3 to 5 in $30 \%$. Five hundred and three (18.3\%) patients died in hospital within 30 days, 101 (20.1\%) within 1 day of admission. Initial empirical antibiotics were in accordance with local CAP guidelines in 1478 $(55.5 \%)$ patients and were administered intravenously in $712(65 \%), 603(74 \%)$ and $743(90 \%)$ patients with CURB65 scores 0 to 1,2 and 3 to 5 respectively. Within 4 hours of admission, a chest $x$-ray was obtained in $83 \%$ of patients and the first dose of antibiotics was administered in $58 \%$.

Conclusions The burden of CAP is high. Efforts should be directed at improving adherence to local CAP guidelines and specific processes of care.

\section{INTRODUCTION}

In conjunction with the updated British Thoracic Society (BTS) guidelines for the management of community acquired pneumonia (CAP) in adults, ${ }^{1}$ the first national BTS audit of adult CAP was conducted as part of the BTS Audit Programme.

\section{METHODS}

An audit tool was developed as part of the BTS CAP guidelines. In autumn 2009, BTS members and registered users of the BTS Audit System were invited to participate in the audit which sought to capture data relating to acutely ill adults admitted to hospitals in the UK and treated for CAP within the period 1 December 2009 and 31 January 2010. Inclusion criteria were immunocompetent adults aged $>16$ years, new infiltrates on chest x-ray, symptoms and signs of a lower respiratory tract infection and no hospital admissions in the previous 10 days. Data entry using the web-based audit tool closed in May 2010.
Data were analysed using SPSS Version $16 . \chi^{2}$ or Fisher exact tests were used to assess differences in proportions. Where applicable, logistic regression was performed to identify factors associated with clinical outcome measures such as inpatient (IP) mortality, and multivariable regression was applied to adjust for disease severity (using the CURB65 score).

\section{RESULTS}

A total of 2749 submissions were received from 64 institutions; 8 submissions were excluded due to inconsistent data, leaving 2741 cases for analysis. The median age of patients was 76 years (range 16-105), one-third were aged $<65$ years and $25 \%$ $(n=687)$ were aged $\geq 85$ years. Details of comorbid illnesses are shown in table $\mathrm{w} 1$ in the online supplement. The CURB65 score was $0-1$ in $40 \%$ of patients, 2 in $30 \%$ and $3-5$ in $30 \%$.

Median length of stay was 5 days and median time to death was 5 days. Of $503(18.3 \%)$ patients who died in hospital within 30 days, 101 (20.1\%) died within 1 day of admission and $62.5 \%$ died within 7 days. The 30-day IP mortality in patients aged $\geq 85$ years was $30.6 \%$ (210/686 patients) (see table w2 in online supplement). Of $192(7 \%)$ patients admitted to intensive care, $97(51 \%)$ had CURB65 scores of $\geq 3,53 \%$ were aged $65-84$ years and $8 \%$ were aged $\geq 85$ years.

\section{Initial antibiotic use and processes of care}

Adherence to local antibiotic guidelines was poor but increased significantly as the CURB65 score increased (OR 1.2, 95\% CI 1.1 to 1.3; table 1). There was no association between adherence to local antibiotic guidelines and 30-day IP mortality after adjusting for disease severity (OR $0.9,95 \% \mathrm{CI}$ 0.8 to 1.2$)$.

Intravenous antibiotics were administered in $712(65 \%), 603(74 \%)$ and $743(90 \%)$ patients with CURB65 scores $0-1,2$ and $3-5$, respectively. A $\beta$-lactam was given in 2060 (75\%) patients and a macrolide in 1641 (60\%) patients (see table w3 in online supplement). A macrolide/ $\beta$-lactam combination was given in 557 (51\%) patients with CURB65 scores $0-1$, while a $\beta$-lactam alone was given in $176(21 \%)$ and $154(19 \%)$ patients with CURB65 scores 2 and 3-5, respectively.

A chest $\mathrm{x}$-ray was performed within $4 \mathrm{~h}$ of admission in 2115 (83\%) of 2543 patients (see table w4 in online supplement), which was reviewed in $80 \%$ of 2531 patients prior to administration of antibiotics. Of 472 patients who died in hospital within 30 days, $283(60 \%)$ received antibiotics 
Table 1 Adherence to local CAP antibiotic guidelines

\begin{tabular}{llc}
\hline CURB65 score & No. of patients & $\begin{array}{l}\text { Initial antibiotics in line with } \\
\text { local CAP guidelines, } \mathbf{n}(\%)\end{array}$ \\
\hline $0-1$ & 1059 & $522(49)$ \\
2 & 800 & $443(55)$ \\
$3-5$ & 809 & $517(64)$ \\
All & 2668 & $1482(56)$ \\
\hline
\end{tabular}

within $4 \mathrm{~h}$ of admission. Overall, 58\% of patients received the first dose of antibiotics within $4 \mathrm{~h}$ of admission (Table 2).

\section{Use of CPAP and NIV}

Of 52 patients who received continuous positive airways pressure (CPAP) support, 30-day IP mortality was $46 \%$ and 38 (73\%) were admitted to an intensive care unit (ICU). Non-invasive ventilation (NIV) was applied in 179 patients; 30-day IP mortality was $43 \%$ and 77 (43\%) patients were admitted to ICU. The proportion of patients on NIV with underlying chronic obstructive pulmonary disease was similar regardless of ICU admission status; 43\% (33/77) were admitted to the ICU and $47 \%$ were not admitted to the ICU.

\section{DISCUSSION}

This is the largest UK audit of the management of CAP in adults and thus provides a description of the current management of CAP in the UK. The proportions of patients in low, moderate and high severity groups were similar to UK cohort studies. ${ }^{2}$ However, mortality was higher. This difference may be due to the older age profile of patients in the audit.

Most units have local CAP guidelines which are based on BTS guidelines. However, adherence to local CAP guidelines was poor (50-70\% depending on CAP severity), and only $58 \%$ of patients received their first antibiotic within $4 \mathrm{~h}$ of hospital admission despite over $80 \%$ of patients having had a chest x-ray within $4 \mathrm{~h}$. Both the time to first antibiotic and adherence to CAP guidelines have been associated with clinical outcomes. ${ }^{3} 4$ Efforts to improve these aspects of care are therefore warranted.

Intravenous antibiotics and dual therapy were overused in patients who did not have high severity CAP. Broad spectrum intravenous antibiotics have been implicated as drivers of nosocomial infections. Efforts to reduce the time to first antibiotic should not be at the expense of the appropriate choice (and route) of antibiotics.

A surprising number of patients received NIV and CPAP support outside an ICU setting. There are no robust data to
Table 2 Time from admission to first dose of antibiotics $(n=2477)$

\begin{tabular}{|c|c|c|c|}
\hline \multirow[b]{2}{*}{ CURB65 score } & \multicolumn{3}{|c|}{ Time to first antibiotics } \\
\hline & $<4 \mathrm{~h}$ & $4-8 h$ & $>8 \mathrm{~h}$ \\
\hline $0-1$ & $566(58)$ & $201(21)$ & $210(21)$ \\
\hline 2 & $390(52)$ & $182(24)$ & $177(24)$ \\
\hline $3-5$ & $478(63)$ & 144 (19) & $129(17)$ \\
\hline All & $1434(58)$ & $527(21)$ & $516(21)$ \\
\hline
\end{tabular}

indicate that NIV or CPAP are effective in CAP, while some data-particularly in relation to CPAP-suggest potential harm. ${ }^{5}$ These forms of support should be used with care in patients with CAP.

Limitations included no measure of completeness of or potential bias in case ascertainment during the audit period and absence of data on frequency of nursing home residence and 'do not resuscitate' orders.

\section{Conclusion}

The burden of CAP is high. Efforts should be directed at improving specific processes of care including adherence to local CAP guidelines.

Acknowledgements This audit would not have been possible without the hard work of the BTS audit team, particularly Sally Welham, Kerry Reid, Chris Routh and Christine Bucknall. We also thank all those who participated in the audit.

\section{Competing interests None.}

Provenance and peer review Not commissioned; internally peer reviewed.

\section{REFERENCES}

1. Lim WS, Baudouin SV, George RC, et al. BTS guidelines for the management of community acquired pneumonia in adults: update 2009. Thorax 2009;64(Suppl 3): iii1-55.

2. Lim WS, van der Eerden MM, Laing R, et al. Defining community acquired pneumonia severity on presentation to hospital: an international derivation and validation study. Thorax 2003;58:377-82.

3. Meehan TP, Weingarten SR, Holmboe ES, et al. A statewide initiative to improve the care of hospitalized pneumonia patients: the Connecticut Pneumonia Pathway Project. Am J Med 2001;111:203-10.

4. McCabe C, Kirchner C, Zhang H, et al. Guideline-concordant therapy and reduced mortality and length of stay in adults with community-acquired pneumonia: playing by the rules. Arch Intern Med 2009;169:1525-31.

5. Delclaux C, L'Her E, Alberti C, et al. Treatment of acute hypoxemic nonhypercapnic respiratory insufficiency with continuous positive airway pressure delivered by a face mask: a randomized controlled trial. JAMA 2000;284:2352-60. 